\title{
Attentional demands associated with augmented visual feedback during quiet standing
}

\author{
Krzysztof Kręcisz ${ }^{\text {Corresp., } 1}{ }^{1}$, Michał Kuczyński ${ }^{2}$ \\ ${ }^{1}$ Faculty of Physical Education and Physiotherapy, Opole University of Technology, Opole, Poland \\ 2 Faculty of Physiotherapy, University School of Physical Education in Wroclaw, Wroclaw, Poland \\ Corresponding Author: Krzysztof Kręcisz \\ Email address: k.krecisz@po.edu.pl
}

To investigate how additional visual feedback (VFB) affects postural stability we compared 20-sec center-of-pressure (COP) recordings in two conditions: without and with the VFB. Seven healthy adult subjects performed 10 trials lasting 20 seconds in each condition. Simultaneously, during all trials the simple auditory reaction time (RT) was measured. Based on the COP data, the following sway parameters were computed: standard deviation (SD), mean speed (MV), sample entropy (SE), and mean power frequency (MPF). The RT was higher in the VFB condition $(p<0.001)$ indicating that this condition was attention demanding. The VFB resulted in decreased SD and increased SE in both the medial-lateral $(\mathrm{ML})$ and anterior-posterior (AP) planes $(\mathrm{p}<.001)$. These results account for the efficacy of the VFB in stabilizing posture and in producing more irregular COP signals which may be interpreted as higher automaticity and/or larger level of noise in postural control. The MPF was higher during VFB in both planes as was the MV in the AP plane only $(p<0.001)$. The latter data demonstrate higher activity of postural control system that was caused by the availability of the set-point on the screen and the resulting control error which facilitate and speed up postural control. 


\section{Attentional demands associated with augmented visual feedback during quiet standing}

2 Krzysztof Kręcisz ${ }^{1 *}$, Michał Kuczyński

$3{ }^{1}$ Faculty of Physical Education and Physiotherapy, Opole University of Technology, Poland

$4{ }^{2}$ Faculty of Physiotherapy, University School of Physical Education in Wroclaw, Wroclaw,

5 Poland

6 *Corresponding author:

7 Krzysztof Kręcisz

8 Faculty of Physical Education and Physiotherapy

9 Opole University of Technology

10 ul. Prószkowska 76, 45-758

11 Opole, Poland

12 E-mail: k.krecisz@po.edu.pl 


\section{Abstract}

15 To investigate how additional visual feedback (VFB) affects postural stability we compared 20-

16 sec center-of-pressure (COP) recordings in two conditions: without and with the VFB. Seven

17 healthy adult subjects performed 10 trials lasting 20 seconds in each condition. Simultaneously, 18 during all trials the simple auditory reaction time (RT) was measured. Based on the COP data,

19 the following sway parameters were computed: standard deviation (SD), mean speed (MV),

20 sample entropy (SE), and mean power frequency (MPF). The RT was higher in the VFB

21 condition $(\mathrm{p}<0.001)$ indicating that this condition was attention demanding. The VFB resulted in

22 decreased SD and increased SE in both the medial-lateral (ML) and anterior-posterior (AP)

23 planes $(\mathrm{p}<.001)$. These results account for the efficacy of the VFB in stabilizing posture and in

24 producing more irregular COP signals which may be interpreted as higher automaticity and/or

25 larger level of noise in postural control. The MPF was higher during VFB in both planes as was

26 the MV in the AP plane only $(\mathrm{p}<0.001)$. The latter data demonstrate higher activity of postural

27 control system that was caused by the availability of the set-point on the screen and the resulting

28 control error which facilitate and speed up postural control. 


\section{Introduction}

The contribution of visual control of balance while standing increases significantly with additional visual feedback (VFB) implemented through the conscious control of the center of pressure displacements under the feet (COP) (Litvinenkova \& Hlavacka, 1973). A positive effect of VFB has been shown in many studies (Litvinenkova \& Hlavacka, 1973; Takeya, 1976; Rougier, 2003) indicating a reduction of the centre of gravity motions with associated increase in muscular activity. However, there are also the investigations that do not confirm such influence. Results of Danna-Dos-Santos et al., (2008) indeed demonstrated that participants were unable to decrease sway amplitude when presented with visual feedback, whereas in Boudrahem \& Rougier, (2009) it has been shown that only $69 \%$ of subjects were able to use additional visual feedback to reduce COP displacements. Ambiguous findings are also reports of attempts to apply VFB technique for rehabilitation. In some studies, the effects appear quite positive (Shumway-Cook, Anson \& Haller, 1988; Sihvonen, Sipilä \& Era, 2004; Cheng et al., 2004; Ledebt et al., 2005; Sayenko et al., 2010), whereas others should be considered as scarce (C et al., 1984; Walker, Brouwer \& Culham, 2000; Geiger et al., 2001). The sources of these discrepancies may be inherent in the way of presenting feedback (insufficient scale display of COP on the screen (Vuillerme, Bertrand \& Pinsault, 2008) and/or delay of the signal on the screen (Rougier, 2004; van den Heuvel et al., 2009)), but also a high cognitive demands associated with learning a new task (Wulf \& Shea, 2002; van Vliet \& Wulf, 2006). Also, many studies have provided evidence that there are significant attentional requirements for postural control (Woollacott \& Shumway-Cook, 2002). Further, the attentional demand associated with postural control can be modified by the sensory context (Vuillerme, Isableu \& Nougier, 2006). A fuller understanding of postural control mechanisms through the conscious control of the center of pressure displacements under the feet allows for a more in-depth diagnosis of certain pathological conditions, and can also be important in training the balance of both patients and athletes (Szczepańska-Gieracha et al., 2016).

Taken together, there is still no consensus as to how vertical posture is controlled when the participants are presented with visual feedback from the actual position of their COP. The predominant view is that attentional resources must be involved due to the larger complexity of the VFB as compared to quiet stance (Lakhani \& Mansfield, 2015), however little is known in 
60 what way these resources are used and whether their shifts facilitate or interfere with maintaining

61 balance. To better elucidate the underlying mechanisms, it seems crucial to compare the COP

62 measures during VFB and quiet stance with simultaneously recorded reaction time task. While

63 the traditional sway measures account for postural performance, of special interest is sway

64 entropy which quantifies the attentional investment in postural control (Roerdink, Hlavackova \&

65 Vuillerme, 2011).

66 Therefore, this study aims to answer the question whether and how the postural task with

67 additional visual feedback requires more attentional demands in young adults. Therefore, we examined reaction times during VFB and change of the amount and structure of COP time series.

69

\section{Methods}

Seven young students participated in the study (mean age (SD): 22.9 (1.1) years; range: 22-25 years, 3 females, 4 males). All subjects were healthy and did not undergo any disease that might affect the balance system. They gave their written informed consent to the procedure and were naive as to the purpose of the experiment. The study was approved by the Senate Ethics Committee for Research at the University School of Physical Education in Wroclaw. Written informed consent was obtained from all participants.

Data were collected as previously described in (Simoneau, Bégin \& Teasdale, 2006; Vuillerme, Isableu \& Nougier, 2006). Specifically, postural stability was assessed on a force plate (AccuSway, AMTI, Watertown, MA) in front of a computer monitor positioned at eye level at a distance of $1 \mathrm{~m}$. Participants were asked to perform two different dual tasks. In the reference condition (REF), they were asked to sway as little as possible fixating a white sign on the screen and simultaneously perform a probe-reaction time (RT) task. The RT task consisted of responding as rapidly as possible to an unpredictable auditory stimulus by pressing a handheld button. Eight reaction time stimuli were presented within each $20 \mathrm{~s}$ trial. During second condition (VFB), the COP position was displayed as a spot (diameter of about $4 \mathrm{~mm}$ ) on the monitor and the subjects were instructed to keep the spot inside the circle (diameter of about 5 $\mathrm{mm}$ ) on the monitor and simultaneously perform RT task. The ratio between the real displacements of the COP and their display on the 19-inch screen was twofold for both the anterior-posterior (AP) and medial-lateral (ML) planes. The AP and ML displacements of the 
91 COP were represented on the screen from top to bottom and from left to right, respectively.

92 Postural tasks were the primary tasks, and the subjects were asked to treat it as a priority.

93 Subjects stood in the position as follows: $17 \mathrm{~cm}$ between heel centers, with an angle of $14^{\circ}$

94 between the long axes of the feet (McIlroy \& Maki, 1997). Ten trials for each condition (lasting

95 20-second each) were presented in pseudo-random (balanced) order.

97 Data were recorded at sampling frequency $50 \mathrm{~Hz}$. The instantaneous center of foot pressure was 98 calculated from the recorded ground reaction forces in the medial-lateral and anterior-posterior 99 plane separately. The raw COP data were not digitally filtered. Postural balance was evaluated

100 by following parameters based on the COP time-series: standard deviation (SD), mean speed 101 (MV), mean power frequency (MPF) (Prieto et al., 1996; Duarte \& Freitas, 2010) and sample 102 entropy (SE) (Richman \& Randall Moorman, 2000). The SD, MV measures postural 103 performance, with lower values of these parameters indicating better performance and MPF 104 reveals postural strategy. SD, MV and MPF were computed using MATLAB codes available at 105 (Duarte \& Freitas, 2010). SD is the dispersion of COP displacement from the mean position 106 during a trial duration, MV was calculated as the total COP displacement divided by trial 107 duration and MPF is the mean spectral power frequency of the signal estimated up to $25 \mathrm{~Hz}$ 108 range. The power spectral density of the detrended COP data was estimated by the Welch 109 periodogram method. SE indexes the regularity or predictability of a time-series. Increased 110 values of sample entropy, which indicate larger irregularity of the COP, has been attributed to a 111 reduced amount of attention invested in posture (Roerdink, Hlavackova \& Vuillerme, 2011).

112 Input parameters for estimating the sample entropy were based on the median value of the 113 relative error (Lake et al., 2002) resulting in the selection of pattern length $\mathrm{m}=2$ and error 114 tolerance $\mathrm{r}=0.08$ and 0.05 as optimal parameters for ML and AP time series (normalized to unit 115 variance) respectively of all subjects and trials. SE was computed using MATLAB script 116 available at www.physionet.org. RT (in milliseconds) helped for determining the attentional

117 demand associated with postural control and was defined as the time interval between the 118 presentation of the auditory stimulus and the subjects' pressing the handheld button (Abernethy, 119 1988). 
121 A linear mixed-effects model was used to test the effect of VFB on RT and COP indices. Trial,

122 feedback (no feedback vs. additional visual feedback) and their interaction were subjected as

123 fixed factors. The effect of trial was chosen as fixed factor to account for any potential fatigue

124 and/or learning effects. Participants were included as a random intercept to take dependency

125 (correlation structure) in the data into the account (Kuznetsov et al., 2015; Boisgontier et al.,

126 2017). Due to skewed distributions, we used log10-transformed data. The level of significance

127 was set at $\mathrm{P}<0.05$. Random intercept models take into account the dependence of repeated trials

128 and have substantial advantages over repeated measures ANOVA. All analyses were performed

129 using free and open software JAMOVI 0.8.2.2 with GAMLj module (retrieved from

130 https://www.jamovi.org).

131

132 Results

133 RT was higher in the VFB condition (fixed effects ANOVA, F(1,1094)=96.89, p<0.001). No

134 trial main effect $(\mathrm{F}(9,1094)=1.32, \mathrm{p}=0.219)$ or feedback $\mathrm{x}$ trial interaction $(\mathrm{F}(9,1094)=1.16$,

$135 \mathrm{p}=0.317$ ) showed statistical significance. The distributions of the RT are shown in Figure 1.

136

137 Figure 1 about here

138

139 For SD there were no interaction feedback x trial effects for both $\operatorname{ML}(F(9,114)=0.92, p=0.507)$

140 and AP planes $(\mathrm{F}(9,114)=1.82, \mathrm{p}=0.072)$. SD was lower in the VFB condition for both ML

$141(\mathrm{~F}(1,114)=68.24, \mathrm{p}<0.001)$ and AP planes $(\mathrm{F}(1,114)=62.47, \mathrm{p}<0.001)$; there was no significant

142 main effect of trial $(\mathrm{ML}: \mathrm{F}(9,114)=1.13, \mathrm{p}=0.347$; $\mathrm{AP}: \mathrm{F}(9,114)=0.95, \mathrm{p}=0.483)$.

143 For MV there were no interaction feedback $x$ trial effects for both $\operatorname{ML}(F(9,114)=0.96, p=0.479)$

144 and AP planes $(\mathrm{F}(9,114)=1.40, \mathrm{p}=0.198)$. MV was higher in the VFB condition for AP plane

$145(\mathrm{~F}(1,114)=15.08, \mathrm{p}<0.001)$ and showed no change in the $\mathrm{ML}$ plane $(\mathrm{F}(1,114)=0.71, \mathrm{p}=0.402)$;

146 there was no significant main effect of trial $(\mathrm{ML}: \mathrm{F}(9,114)=0.85, \mathrm{p}=0.570 ; \mathrm{AP}: \mathrm{F}(9,114)=0.74$, $147 \mathrm{p}=0.675)$.

148 For SE there were no interaction feedback $x$ trial effects for both $\operatorname{ML}(\mathrm{F}(9,114)=0.94, \mathrm{p}=0.490)$

149 and AP planes $(\mathrm{F}(9,114)=1.07, \mathrm{p}=0.393)$. SE was higher in the VFB condition for both ML

$150 \quad(\mathrm{~F}(1,114)=78.61, \mathrm{p}<0.001)$ and AP planes $(\mathrm{F}(1,114)=74.83, \mathrm{p}<0.001)$; there was no significant

151 main effect of trial $(\mathrm{ML}: \mathrm{F}(9,114)=1.61, \mathrm{p}=0.120 ; \mathrm{AP}: \mathrm{F}(9,114)=1.60, \mathrm{p}=0.123)$. 
152 For MPF there were no interaction feedback x trial effects for both $\operatorname{ML}(F(9,114)=0.98, p=0.463)$

153 and AP planes $(F(9,114)=0.846, p=0.575)$. MPF was higher in the VFB condition for both ML

$154(\mathrm{~F}(1,114)=68.49, \mathrm{p}<0.001)$ and AP planes $(\mathrm{F}(1,114)=99.99, \mathrm{p}<0.001)$; there was no significant

155 main effect of trial $(\mathrm{ML}: \mathrm{F}(9,114)=0.813$, $\mathrm{p}=0.605$; AP: $\mathrm{F}(9,114)=0.50, \mathrm{p}=0.827)$.

156

157 The distributions of the COP parameters are shown in the Figure 2.

158

159 Figure 2 about here

160

161 All analyses are available for download using the Open Science Framework: https://osf.io/mptkr/ 162

163

Discussion

164 The purpose of this study was to determine whether and how the postural task with additional

165 visual feedback requires additional attentional demands in young adults. The results show that:

166 1) VFB condition requires additional attentional demands because reaction times were longer, 2)

167 concurrent visual feedback about postural sway shifts focus of attention not directly to postural

168 control because of increase of SE, 3) the implementation of the VFB task has triggered the need

169 for a change in the postural strategy through a reduction in the amount of sway and increase of

170 MV and MPF.

171 In agreement with our work, the increased COP entropy during visual feedback tasks was

172 reported by (Donker et al., 2008) and (Lakhani \& Mansfield, 2015). They attributed these results

173 to the effect of using the external reference system which is thought to facilitate more automatic

174 control of posture (Wulf, McNevin \& Shea, 2001). Similarly, the increased reaction times in our

175 experiments account for shifting the attention of participants to the task of keeping their COP

176 inside the target on the screen. Focusing significant attentional resources on the latter task took

177 from the attention which is normally used to maintaining postural control which also implies

178 more automaticity in maintaining balance.

179 All participants were able to effectively use the VFB in reducing their sway amplitude, yet this

180 activity was accompanied by the increase in sway frequency. Higher frequency of postural sway

181 has been often reported during dual tasks that led to reduced amount of attention which is 
182 normally involved in postural control. It is argued that increased sway frequency results from

183 increased joint stiffness (Vuillerme \& Vincent, 2006; Bieć et al., 2014). Such an interpretation

184 seems justified based on the relationship between the effective postural stiffness and the

185 frequency of the COP signal that was established by (Winter et al., 1998). In contrast, (Stins,

186 Roerdink \& Beek, 2011) did not find a direct association between postural stiffness and the level

187 of automaticity in controlling posture. However, it is possible that postural tasks with visual

188 feedback have different effects on the distribution of attentional resources than other

189 supplementary cognitive tasks that are apparently not related to posture.

190 The difference lies in the final application of the attention diverted from posture and invested

191 into the supplementary task. In the latter tasks a necessary portion of attentional resources is

192 being withdrawn from the normal postural control and this loss requires compensation or some

193 other form of reinforcement. According to several authors, this is usually accomplished by

194 promoting the more automatic control process (Kuczyński, Szymańska \& Bieć, 2011; Lakhani \&

195 Mansfield, 2015).

196 However, in the former task, attention transferred to the VFB was indirectly reverted and

197 actually supported the process of postural control. Larger reaction times in the VFB indicate that

198 this task is attention demanding and one can speculate that the diverted attention is necessary for

199 the integration of the ancillary visual input with the remaining sensory information. Again,

200 elevated sway frequency seems to irrevocably contribute to this purpose. In fact, the stiffening of

201 postural strategy was suggested as the means to perform the postural exploratory and/or

202 monitoring function which significantly increases with the difficulty of postural tasks (Latash et

203 al., 2003). This exploratory function of sway is ceaselessly active, even during conscious control

204 of posture, and is thought to have random bearing. A certain level of randomness of spontaneous

205 sway is inherent because of its unconscious origin. However, an additional and quite substantial

206 uncertainty in this signal may develop from the conscious action of participants using the visual

207 error to correct their position inside the target on the screen. Although the purpose of action is

208 conscious, its timing and magnitude are not, and the two latter factors depend heavily on the

209 sensorimotor abilities and performance of the subjects. In other words, increased sway entropy

210 observed during VFB may be not only the consequence of a more automatic control of posture

211 but also the reflection of increased uncertainty in performance. This would be in agreement with 
212 (Morrison, Hong \& Newell, 2007) who found that subjects who voluntarily produced random

213 sway motions exhibited higher COP entropy as compared to standing still. In a similar vein

214 (Borg \& Laxaback, 2010) postulated that higher entropy may be interpreted as an inability in

215 some circumstances to exert effective attentive control.

\section{Conclusions}

217 In conclusion, the VFB is effective in enhancing and improving postural performance. This

218 benefit is associated with increased sensorimotor activity which effect on humans, depending on

219 circumstances, may be different. VFB has higher attentional demands as compared to normal

220 stance. VFB increases irregularity and entropy of sway, still presented results seem insufficient

221 to disentangle the role of elevated automaticity or noisiness in these changes.

222

223 References

224 Abernethy B. 1988. Dual-task methodology and motor skills research: Some applications and 225 methodological constraints. Journal of Human Movement Studies 14:101-132.

226 Boisgontier MP., Cheval B., Chalavi S., van Ruitenbeek P., Leunissen I., Levin O., Nieuwboer

227

228

229

230

231

232

233

234

235

236

237

238

239

240

241

A.. Swinnen SP. 2017. Individual differences in brainstem and basal ganglia structure predict postural control and balance loss in young and older adults. Neurobiology Aging 50:47-59. DOI: 10.1016/j.neurobiolaging.2016.10.024

Borg FG., Laxaback G. 2010. Entropy of balance - some recent results. Journal of NeuroEngineering and Rehabilitation 7:38. DOI: 10.1186/1743-0003-7-38.

Boudrahem S., Rougier PR. 2009. Relation between postural control assessment with eyes open and centre of pressure visual feedback effects in healthy individuals. Experimental Brain Research 195:145-152. DOI: 10.1007/s00221-009-1761-1.

Cheng PT., Wang CM., Chung CY., Chen CL. 2004. Effects of visual feedback rhythmic weightshift training on hemiplegic stroke patients. Clinical Rehabilitation 18:747-753. DOI: 10.1191/0269215504cr778oa.

Danna-Dos-Santos A., Degani AM., Zatsiorsky VM., Latash ML. 2008. Is voluntary control of natural postural sway possible? Journal of Motor Behavior 40:179-185. DOI: 10.3200/JMBR.40.3.179-185.

Donker SF., Ledebt A., Roerdink M., Savelsbergh GJP., Beek PJ. 2008. Children with cerebral 
242

243

244

245

246

247

248

249

250

251

252

253

254

255

256

257

258

259

260

261

262

263

264

265

266

267

268

269

270

271

272

palsy exhibit greater and more regular postural sway than typically developing children. Experimental Brain Research 184:363-370. DOI: 10.1007/s00221-007-1105-y.

Duarte M., Freitas SMSF. 2010. Revision of posturography based on force plate for balance evaluation. Revista Brasileira de Fisioterapia 14:183-192. DOI: 10.1590/S141335552010000300003.

Geiger R a., Allen JB., O'Keefe J., Hicks RR. 2001. Balance and mobility following stroke: effects of physical therapy interventions with and without biofeedback/forceplate training. Physical therapy 81:995-1005. DOI: 11276182.

van den Heuvel MRC., Balasubramaniam R., Daffertshofer A., Longtin A., Beek PJ. 2009. Delayed visual feedback reveals distinct time scales in balance control. Neuroscience Letters 452:37-41. DOI: 10.1016/j.neulet.2009.01.024.

Kuczyński M., Szymańska M., Bieć E. 2011. Dual-task effect on postural control in high-level competitive dancers. Journal of Sports Sciences 29:539-45. DOI: 10.1080/02640414.2010.544046.

Kuznetsov NA., Luberto CM., Avallone K., Kraemer K., McLeish AC., Riley MA. 2015. Characteristics of postural control among young adults with asthma. Journal of Asthma 52:191-7. DOI: 10.3109/02770903.2014.954290.

Lake DE., Richman JS., Griffin MP., Moorman JR. 2002. Sample entropy analysis of neonatal heart rate variability. American Journal of Physiology - Regulatory, Integrative and Comparative Physiology 283:R789-R797. DOI: 10.1152/ajpregu.00069.2002.

Lakhani B., Mansfield A. 2015. Visual feedback of the centre of gravity to optimize standing balance. Gait and Posture 41:499-503. DOI: 10.1016/j.gaitpost.2014.12.003.

Latash ML., Ferreira SS., Wieczorek SA., Duarte M. 2003. Movement sway: changes in postural sway during voluntary shifts of the center of pressure. Experimental Brain Research 150:314-324. DOI: 10.1007/s00221-003-1419-3.

Ledebt A., Becher J., Kapper J., Rozendaal RM., Bakker R., Leenders IC., Savelsbergh GJP. 2005. Balance Training with Visual Feedback in Children with Hemiplegic Cerebral Palsy: Effect on Stance and Gait. Motor Control 9:459-468. DOI: 10.1123/mcj.9.4.459.

Litvinenkova V., Hlavacka F. 1973. The visual feedback gain influence upon the regulation of the upright posture in man. Agressologie 14:95-99.

McIlroy WE., Maki BE. 1997. Preferred placement of the feet during quiet stance: Development 
273

274

275

276

277

278

279

280

281

282

283

284

285

286

287

288

289

290

291

292

293

294

295

296

297

298

299

300

301

302

303

of a standardized foot placement for balance testing. Clinical Biomechanics 12:66-70. DOI: 10.1016/S0268-0033(96)00040-X.

Morrison S., Hong SL., Newell KM. 2007. Inverse relations in the patterns of muscle and center of pressure dynamics during standing still and movement postures. Experimental Brain Research 181:347-358. DOI: 10.1007/s00221-007-0928-x.

Prieto TE., Myklebust JB., Hoffmann RG., Lovett EG., Myklebust BM. 1996. Measures of postural steadiness: Differences between healthy young and elderly adults. IEEE Transactions on Biomedical Engineering 43:956-966. DOI: 10.1109/10.532130.

Richman JS., Randall Moorman J. 2000. Physiological time-series analysis using approximate entropy and sample entropy. Am.J.Physiol Heart Circ Physiol 278:H2039-H2049.

Roerdink M., Hlavackova P., Vuillerme N. 2011. Center-of-pressure regularity as a marker for attentional investment in postural control: A comparison between sitting and standing postures. Human Movement Science 30:203-212. DOI: 10.1016/j.humov.2010.04.005.

Rougier P. 2003. Visual feedback induces opposite effects on elementary centre of gravity and centre of pressure minus centre of gravity motions in undisturbed upright stance. Clinical Biomechanics 18:341-349. DOI: 10.1016/S0268-0033(03)00003-2.

Rougier P. 2004. Optimising the visual feedback technique for improving upright stance maintenance by delaying its display: Behavioural effects on healthy adults. Gait and Posture 19:154-163. DOI: 10.1016/S0966-6362(03)00056-0.

Sayenko DG., Alekhina MI., Masani K., Vette AH., Obata H., Popovic MR., Nakazawa K. 2010. Positive effect of balance training with visual feedback on standing balance abilities in people with incomplete spinal cord injury. Spinal Cord 48:886-893. DOI: 10.1038/sc.2010.41.

Shumway-Cook A., Anson D., Haller S. 1988. Postural sway biofeedback: its effect on reestablishing stance stability in hemiplegic patients. Archives of Physical Medicine and Rehabilitation 69:395-400.

Sihvonen SE., Sipilä S., Era PA. 2004. Changes in Postural Balance in Frail Elderly Women during a 4-Week Visual Feedback Training: A Randomized Controlled Trial. Gerontology 50:87-95. DOI: 10.1159/000075559.

Simoneau M., Bégin F., Teasdale N. 2006. The effects of moderate fatigue on dynamic balance control and attentional demands. Journal of NeuroEngineering and Rehabilitation 3:22. 
304

305

306

307

308

309

310

311

312

313

314

315

316

317

318

319

320

321

322

323

324

325

326

327

328

329

330

331

332

333

334

DOI: $10.1186 / 1743-0003-3-22$.

Stins JF., Roerdink M., Beek PJ. 2011. To freeze or not to freeze? Affective and cognitive perturbations have markedly different effects on postural control. Human Movement Science 30:190-202. DOI: 10.1016/j.humov.2010.05.013.

Szczepańska-Gieracha J., Cieślik B., Chamela-Bilińska D., Kuczyński M. 2016. Postural Stability of Elderly People With Cognitive Impairments. American Journal of Alzheimer's Disease \& Other Dementiasr 31:241-246. DOI: 10.1177/1533317515602547.

Takeya T. 1976. A Biofeedback Study of Postural Sway. Psychiatry and Clinical Neurosciences 30:495-504. DOI: 10.1111/j.1440-1819.1976.tb02672.x.

van Vliet P., Wulf G. 2006. Extrinsic feedback for motor learning after stroke: What is the evidence? Disability and Rehabilitation 28:831-840. DOI: 10.1080/09638280500534937.

Vuillerme N., Bertrand R., Pinsault N. 2008. Postural Effects of the Scaled Display of Visual Foot Center of Pressure Feedback Under Different Somatosensory Conditions at the Foot and the Ankle. Archives of Physical Medicine and Rehabilitation 89:2034-2036. DOI: 10.1016/j.apmr.2008.03.017.

Vuillerme N., Isableu B., Nougier V. 2006. Attentional demands associated with the use of a light fingertip touch for postural control during quiet standing. Experimental Brain Research 169:232-236. DOI: 10.1007/s00221-005-0142-7.

Walker C., Brouwer B., Culham E. 2000. Use of visual feedback in retraining balance following acute stroke. Physical Therapy 80:886-895.

Winter DA., Patla AE., Prince F., Ishac M., Gielo-Perczak K. 1998. Stiffness Control of Balance in Quiet Standing. Journal of Neurophysiology 80:1211-1221. DOI: 10.1152/jn.1998.80.3.1211.

Woollacott M., Shumway-Cook A. 2002. Attention and the control of posture and gait: a review of an emerging area of research. Gait \& Posture 16:1-14. DOI: 10.1016/S09666362(01)00156-4.

Wulf G., McNevin N., Shea CH. 2001. The automaticity of complex motor skill learning as a function of attentional focus. The Quarterly Journal of Experimental Psychology A 54:1143-1154. DOI: 10.1080/02724980143000118.

Wulf G., Shea CH. 2002. Principles derived from the study of simple skills do not generalize to complex skill learning. Psychonomic Bulletin and Review 9:185-211. DOI: 
$335 \quad 10.3758 / \mathrm{BF} 03196276$. 
Figure 1

Violin plots of the RT for the REF and the VFB conditions collapsed over trials with mean and standard deviations superimposed.

These plots show full distribution of the data obtained by kernel density estimation. The dot symbol denotes mean, whisker denotes standard deviation.

\section{FEEDBACK $\rightarrow$ REF $\rightarrow$ VFB}

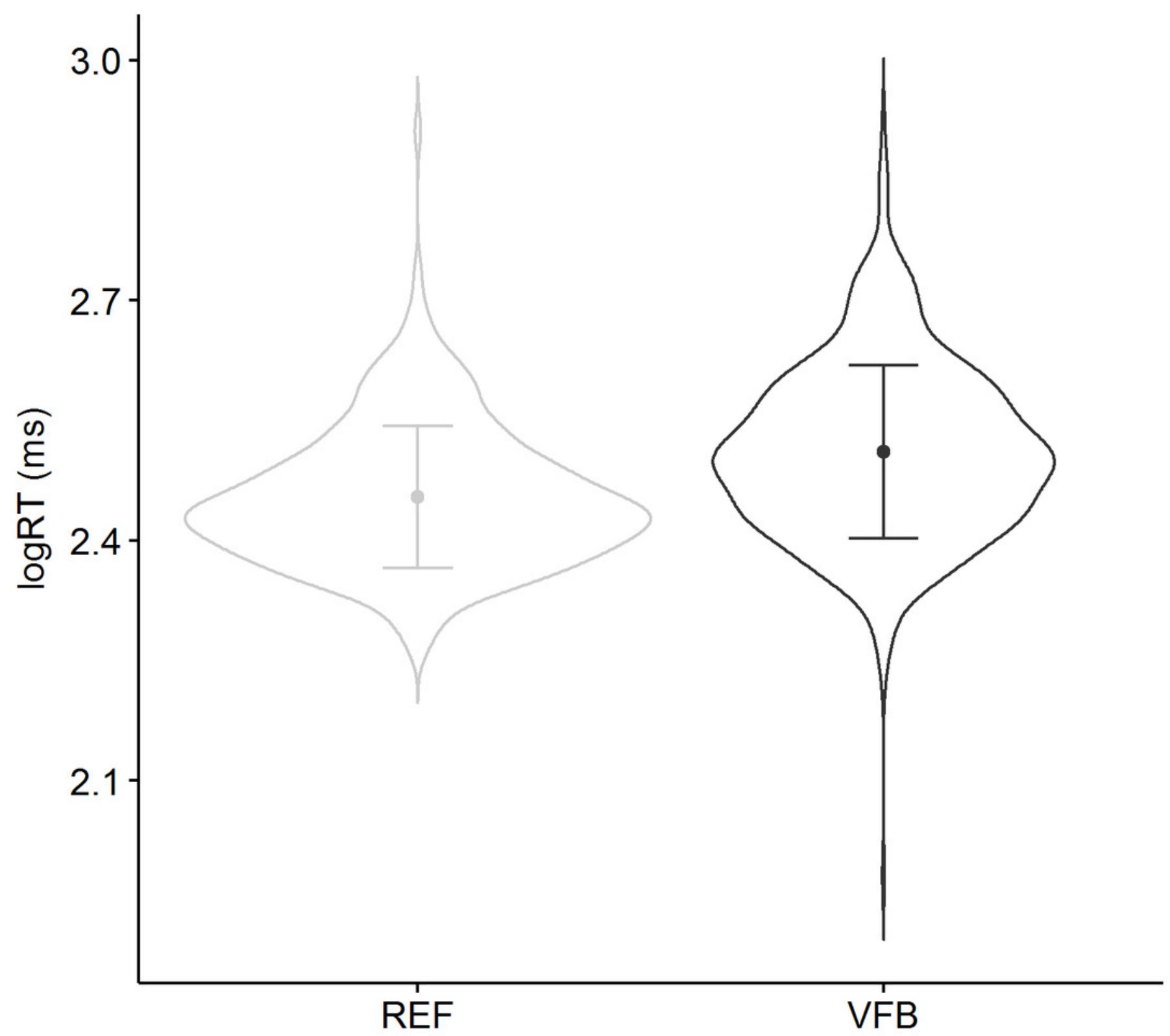


Figure 2

Violin plots of the COP parameters for the REF and the VFB conditions collapsed over trials with and mean \pm standard deviations superimposed: SD - variability, MV - mean speed, SE - sample entropy, MPF - mean power frequency

These plots show full distribution of the data obtained by kernel density estimation. The dot symbol denotes mean, whisker denotes standard deviation.
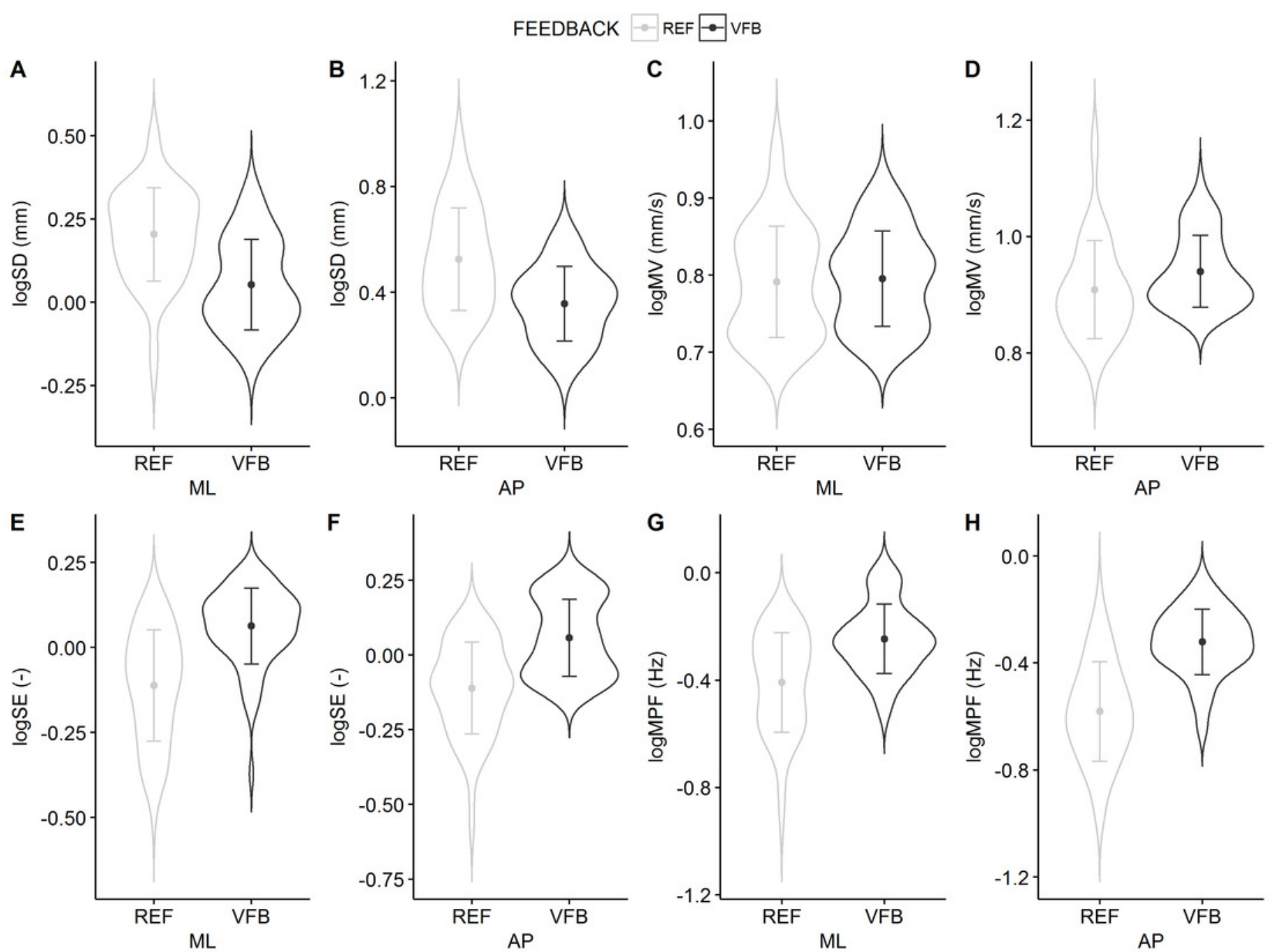